\title{
EVALUATION OF RETRIEVAL ALGORITHMS FOR ICE MICROPHYSICS USING CALIPSO /CLOUDSAT AND EARTHCARE
}

\author{
Hajime Okamoto $^{1 *}$, Kaori Sato ${ }^{1}$, Yuichiro Hagihara ${ }^{1}$, Hiroshi Ishimoto ${ }^{2}$, Anatoli Borovoi ${ }^{3}$, \\ Alexander Konoshonkin ${ }^{3}$, Natalia Kustova ${ }^{3}$ \\ ${ }^{1}$ Research Institute for Applied Mechanics, Kyushu University, Kasuga, Fukuoka 816-8580, Japan, \\ *Email: okamoto@riam.kyushu-u.ac.jp \\ ${ }^{2}$ Meteological Research Institute, Japan Meteorological Agency, Tsukuba, Ibaraki 305-0052, Japan \\ ${ }^{3}$ V.E. Zuev Institute of Atmospheric Optics, Rus. Acad. Sci., Tomsk 634021, Russia
}

\begin{abstract}
We developed lidar-radar algorithms that can be applied to Cloud-Aerosol Lidar and Infrared Pathfinder Satellite Observation (CALIPSO) lidar and CloudSat data to retrieve ice microphysics. The algorithms were the extended version of previously reported algorithm [1] and can treat both of nadir pointing of CALIPSO lidar period and $3^{\circ}$-off-nadir pointing one. We used the scattering data bank produced by the physical optics methods [2] and created lidar look-up tables of quasi-horizontally oriented ice plates (Q2D-plate) for nadir- and off-nadir lidar pointing periods. Then LUTs were implemented in the ice retrieval algorithms.

We performed several sensitivity studies to evaluate uncertainties in the retrieved ice microphysics due to ice particle orientation and shape. It was found that the implementation of orientation of horizontally oriented ice plate model in the algorithm drastically improved the retrieval results in both for nadir- and off-nadir lidar pointing periods. Differences in the retrieved microphysics between only randomly oriented ice model (3D-ice) and mixture of 3D-ice and Q2Dplate model were large especially in off-nadir period, e.g., $100 \%$ in effective radius and one order in ice water content, respectively. And differences in the retrieved ice microphysics among different mixture models were smaller than about $50 \%$ for effective radius in nadir period.
\end{abstract}

\section{INTRODUCTION}

Ice clouds play an essential role in the climate system through their radiative effects. Combination of $94 \mathrm{GHz}$ cloud radar on CloudSat and lidar on CALIPSO was proved to be effective to derive global distribution of ice microphysical properties [1]. Since the lidar wavelength was much smaller than the ice particle size, the lidar backscattering properties (backscattering coefficient and depolarization ratio) depend on particle shape, orientation, size, ice water content (IWC) and laser tilt angle (=off-nadir angle). Currently details of horizontally oriented ice particle models have not been taken into account in the analysis of CloudSat and CALIPSO for the off-nadir period and lidar backscattering properties of oriented ice were assumed to be similar to that of randomly oriented ice. In this study, we developed the new lidar-radar algorithms that can be applied to both periods. And uncertainties of the retrieved ice microphysics were investigated.

\section{ANALYSIS OF CALIPSO LIDAR DATA}

CALIPSO lidar observations showed that specular returns, i.e., large backscattering coefficient (ß), from ice clouds were frequently observed from December 2006 to November 2007 and the period corresponds to the one when the laser direction was close to nadir (off-nadir angle was $0.3^{\circ}$ ) (Fig. 1a) [3]. And fraction of large $\beta\left(>10^{-4}[1 / \mathrm{m} / \mathrm{sr}]\right)$ drastically reduced from December 2007 to November 2008, corresponding to the off-nadir period $\left(3^{\circ}\right)$ (Fig.1b). In these analyses, ice clouds were first identified by the phase discrimination scheme from attenuation and depolarization ratio of CALIPSO lidar observations [3] and only night time data was used.

When the data was sorted by values of $B$ at $532 \mathrm{~nm}$, the depolarization ratio of ice particles for the largest $\beta$ category $\left(>10^{-4}[1 / \mathrm{m} / \mathrm{sr}]\right)$ was 
minimum $(<5 \%)$ when temperature $(\mathrm{T})>-20^{\circ} \mathrm{C}$ for the nadir pointing period (Fig. 2a). And smaller $\beta$ categories generally showed larger depolarization ratio in the same $\mathrm{T}$ range and the depolarization ratio was smaller than $15 \%$.

The depolarization ratio below $-20^{\circ} \mathrm{C}$ during offnadir period was similar to nadir period, while the value above $-20^{\circ} \mathrm{C}$ in the off-nadir period was about $20 \%$, which was larger compared with the value in the nadir period,. These differences between the two periods might attribute to the change in the backscattering properties of oriented ice particles between both periods.

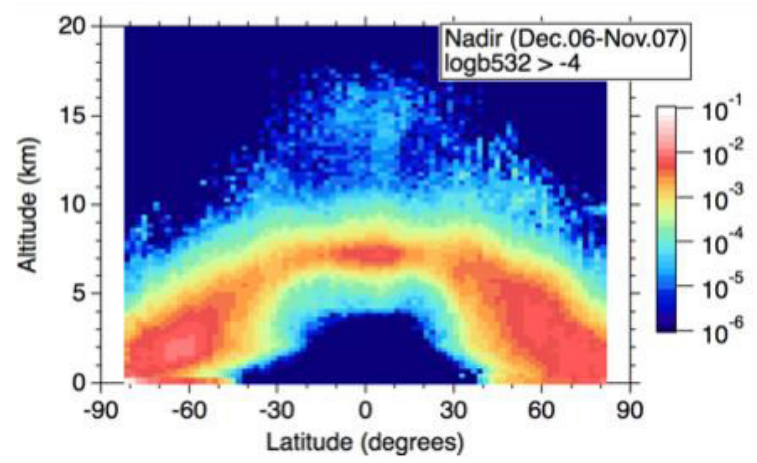

(a)

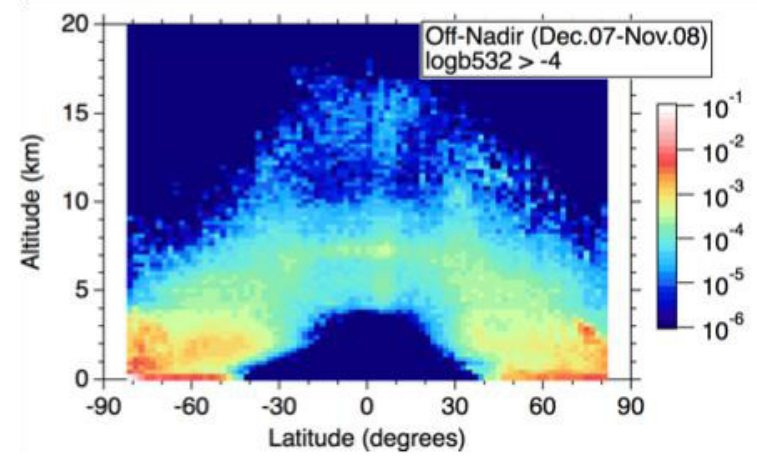

(b)

Fig.1 Height-latitude plot of annual mean cloud fraction for attenuated backscattering coefficient at $532 \mathrm{~nm}>10^{-4}[1 / \mathrm{m} / \mathrm{sr}]$ from CALIPSO lidar observations. (a) Nadir pointing period $\left(0.3^{\circ}\right)$, from December 2006 to December 2007. (b) Offnadir pointing period $\left(3^{\circ}\right)$ from December 2007 to December 2008.

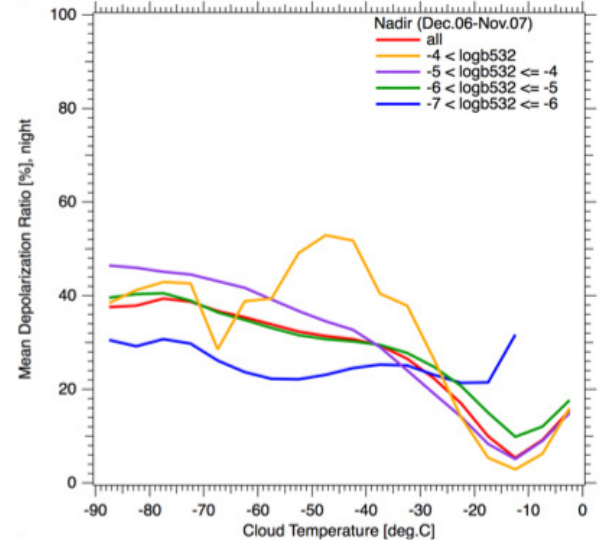

(a)

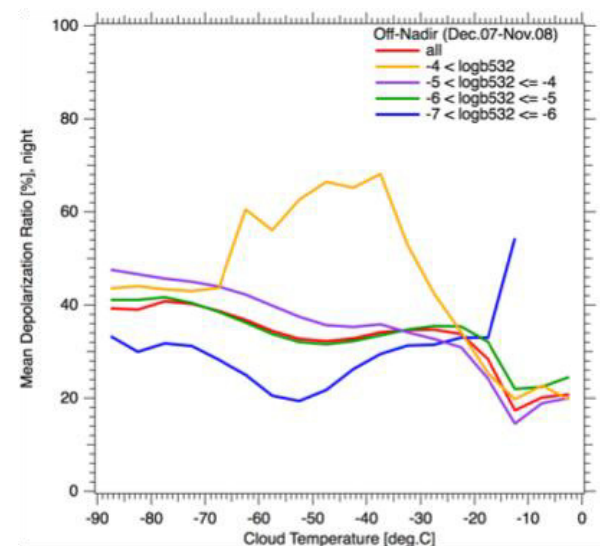

(b)

Fig.2 Annual mean depolarization ratio of ice clouds by CALIPSO lidar. Dependence of temperature on the depolarization ratio sorted by $\beta$ was shown. (a) Nadir period. (b) Off-nadir period.

Next, we examined lidar backscattering properties of quasi-horizontally oriented ice plates with different orientation models and that for randomly oriented ice particles by using physical optics method [2]. The size distribution was assumed to be modified Gamma distribution function with variation of the characteristic radius [1]. The state of orientation of ice plate was characterized by Gamma distribution function with effective distribution width $\theta_{\text {eff }}$ that denotes standard deviation of canting angle of the particles. We considered three values of $\theta$ eff, i.e., $0.1^{\circ}, 0.5^{\circ}$ and $1^{\circ}$ [4]. 3 of Q2D-plate was generally one to three orders of magnitude larger compared with that of 3D-ice for nadir pointing period (Fig.3). B for 
Q2D-plate for the nadir period differed by a factor of 4 among the three $\theta$ eff models.

There were larger differences among Q2D-plate models in off-nadir period compared with those in nadir period. Q2D-plate with $\theta$ eff $=1^{\circ}$ showed the largest $B$ value than other types and Q2D-plate models with $0.1^{\circ}$ and $0.5^{\circ}$ showed smaller $\beta$ than that of $3 \mathrm{D}$-ice in the period. That suggests uncertainties in the ice models might lead to larger uncertainties in the ice microphysical retrievals in off-nadir period.

Since depolarization ratio of Q2D-plate was close to $0 \%$ and that for 3D-ice was $20-40 \%$, mixture of Q2D-plate and 3D-ice model can potentially explain the observed depolarization ratio shown in Fig.2. Therefore the mixture of Q2D-plate and $3 \mathrm{D}$-ice model with variation of the mixing ratio of Q2D-plate was considered in the retrieval algorithms as in [1].
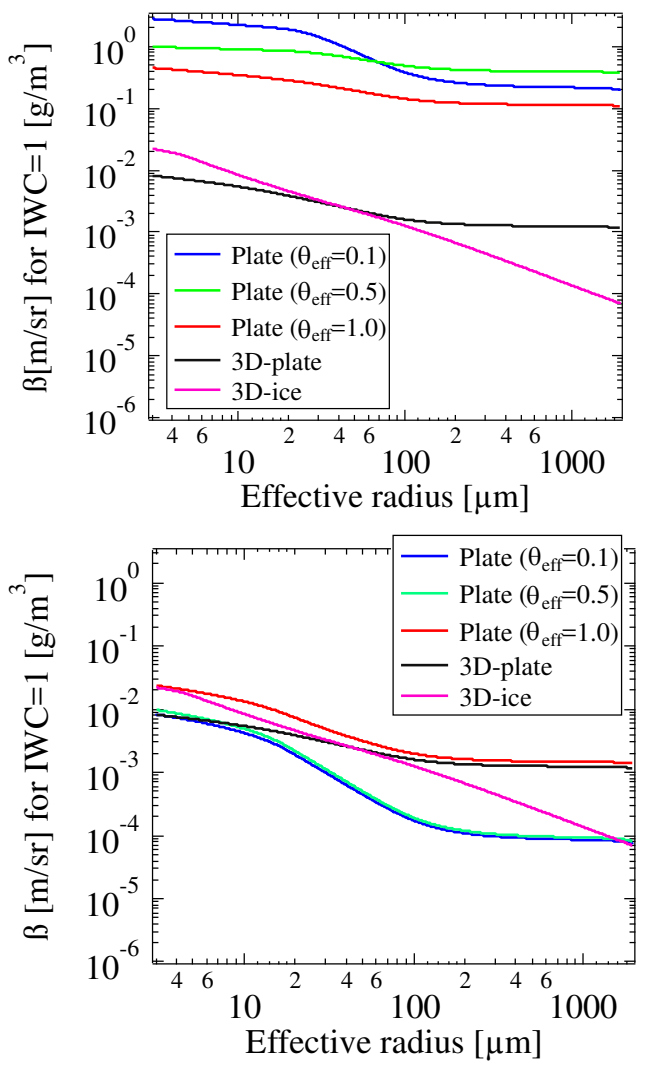

Fig.3 $\beta$ for quasi-horizontally oriented ice plates and randomly oriented ice models as function of effective radius. (a) $B$ for Nadir period and (b) $B$ for off-nadir period. Ice water content was set to be $1 \mathrm{~g} / \mathrm{m}^{3}$.

\section{RETRIEVAL RESULTS OF ICE MICROPHYSCS FROM CALIPSO AND CLOUDSAT}

We estimated look up tables for CALIPSO lidar by the physical optics method described in Section 2 and LUTs for CloudSat radar by the discrete dipole approximation. Then lidar-radar algorithm [1] for ice microphysics with the new look up tables was applied to the CALIPSO and CloudSat data. $B$ and depolarization ratio at $532 \mathrm{~nm}$ and radar reflectivity factor were used and effective radius, IWC and mass mixing ratio of Q2D-plate to the total IWC were retrieved. The results were shown for off-nadir period (Fig 4).

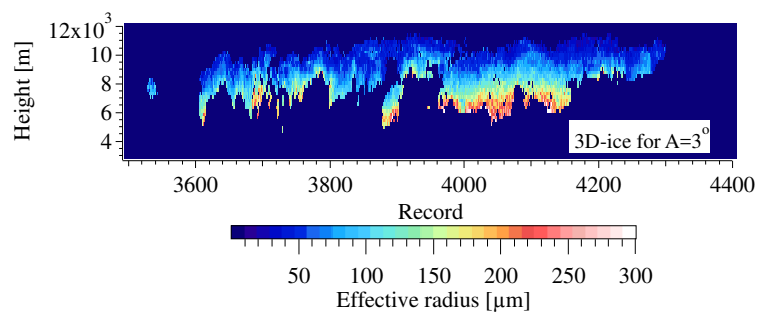

(a)

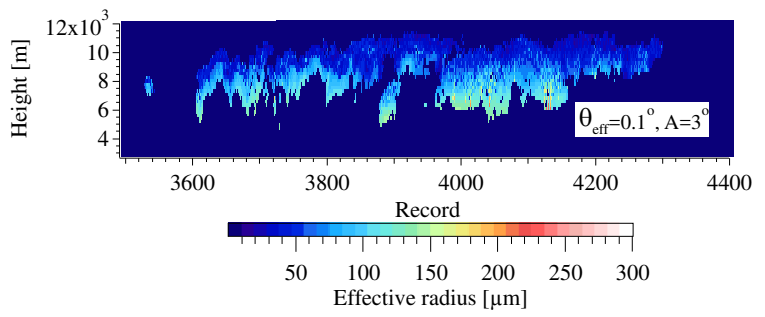

(b)

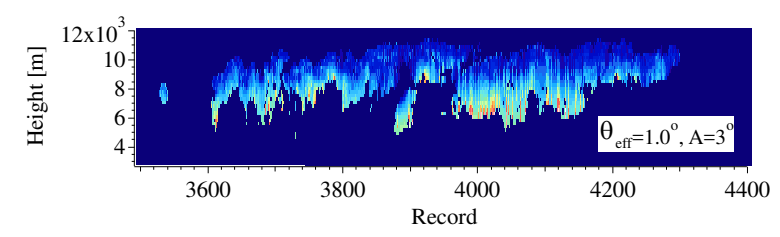

(c)

Fig. 4 Time-height plot of retrieved ice effective radius for $\theta_{\text {eff }}$ to be $0.1^{\circ}, 0.5^{\circ}$ and $1^{\circ}$ by lidarradar algorithms for off-nadir period. (a) results of pure 3D-ice model, (b) results for mixture of 3Dice and Q2D-plate with $\theta$ eff of $0.1^{\circ}$ and (c) results for mixture of 3D-ice and Q2D-plate with $\theta$ eff of $1^{\circ}$.

When only 3D-ice model was considered and Q2D-plate model was not included in the 
retrievals, effective radius was overestimated in the lower part of cloud layers compared with the results for the mixture of 3D-ice and Q2D-plate models. The differences in effective radius and IWC were more than a factor and one order, respectively. This demonstrated the need to take into account the Q2D-plate and the orientation of Q2D-plate in off-nadir period. Differences between the models with $\theta$ eff to be $0.1^{\circ}$ and $1^{\circ}$ were also large, e.g., about $50 \%$ in effective radius and about one order. Thus we considered that the ice orientation would be the major source of retrieval uncertainties in off-nadir period.

Currently there are no retrieval algorithms that can treat $\beta$ at $1064 \mathrm{~nm}$ in addition to $\beta$ at $532 \mathrm{~nm}$. The backscattered color ratio defined as ratio of $B$ at $1064 \mathrm{~nm}$ to $B$ at $532 \mathrm{~nm}$ was investigated. First we used lidar signals from cloud top part of water clouds without dense aerosols or cirrus clouds above to re-calibrate $\beta$ at $1064 \mathrm{~nm}$ for CALIPSO lidar. The color ratio of water clouds was assumed to be unity for the re-calibration of $\beta$ at $1064 \mathrm{~nm}$. Then global analysis of the color ratio of ice particles was conducted. It was shown that the color ratio of ice clouds is about 0.9 with standard deviation of about 0.3. Calculation by physical optics indicated that the color ratio for Q2D-plate was smaller than 1 when effective radius is smaller than $40 \mu \mathrm{m}$ and the value was about 1 for larger particles except for $\theta$ eff to be $0.1^{\circ}$ where the value was much larger than 1 in the nadir period. That for 3D-plates was from 0.7 to 0.8 for the same period. The color ratio for Q2D-plate were larger than 1 for smaller particles and smaller than 1 for large size when $\theta$ eff was assumed to be $0.1^{\circ}$ or $0.5^{\circ}$ for off-nadir period. Therefore the backscatter color ratio might be able to be used to discriminate different orientation models and this could reduce uncertainties in the retrieved ice microphysics.

\section{SUMMARY}

We implemented the new look up tables for quasihorizontally oriented ice plates into the lidar-radar algorithm to retrieve ice microphysics by CALIPSO lidar and CloudSat data. The new algorithms can treat both data obtained in the nadir and the off-nadir laser pointing periods of CALIPSO lidar. We considered standard deviation of canting angle of ice plates from 0.1 to
1 to estimate $\beta$ by the physical optics method for CALIPSO lidar signals. We found mixture of 3Dice and Q2D-plate models could reproduce observed $\beta$ and depolarization ratio for nadir and off-nadir periods.

Sensitivity studies showed that larger differences in the retrieved ice microphysics among Q2Dplate models existed for off-nadir period than those for nadir-period. To reduce uncertainties in the retrieved microphysics, the information of backscattered color ratio of ice might help. JAXAESA joint mission EarthCARE will carry high spectral resolution lidar (ATLID) and Doppler $94 \mathrm{GHz}$ cloud radar in space in 2018. Since ATLID will use off-nadir pointing similar to CALIPSO, it is expected to have similar effects considered in this studies.

\section{ACKNOWLEDGEMENT}

This work was supported in part by the JSPS Kakenhi Grant Number 25247078 and 15K17762 and also supported by the Collaborated Research Program of Research Institute for Applied Mechanics, Kyushu University.

\section{REFERENCES}

[1] Okamoto, H., K. Sato and Y. Hagihara, 2010: Global analysis of ice microphysics from CloudSat and CALIPSO: intercomparison of specular reflection in lidar signals, J. Geophys. Res., 115, D22209, doi:10.1029/2009JD013383.

[2] Borovoi, A., A. Konoshonkin, N. Kustova, H. Okamoto, 2012: Backscattering Mueller matrix for quasi-horizontally oriented ice plates of cirrus clouds: application to CALIPSO signals, Opt. Express, 20(27), 28222-28233,

[3] Yoshida, R., H. Okamoto, Y. Hagihara and H. Ishimoto, 2010: Global analysis of cloud phase and ice crystal orientation from Cloiud-Aerosol Lidar and Infrared Pathfinder Satellite Observation (CALIPSO) data using attenuated backscattering and depolarization ratio, $J$. Geophys. Atmos., 115, D00H32, doi:1029/2009JD012334.

[4] Sassen, K., 1980: Remote sensing of planar ice crystal fall altitudes, J. Meteor. Soc. Japan, 58, 422-429. 